\title{
Method to analyze electromechanical stability of dielectric elastomers
}

\section{Citation}

Zhao, Xuanhe, and Zhigang Suo. 2007. "Method to Analyze Electromechanical Stability of

Dielectric Elastomers." Applied Physics Letters 91 (6): 61921. https://doi.org/10.1063/1.2768641.

\section{Permanent link}

http://nrs.harvard.edu/urn-3:HUL.InstRepos:41467481

\section{Terms of Use}

This article was downloaded from Harvard University's DASH repository, and is made available under the terms and conditions applicable to Other Posted Material, as set forth at http:// nrs.harvard.edu/urn-3:HUL.InstRepos:dash.current.terms-of-use\#LAA

\section{Share Your Story}

The Harvard community has made this article openly available.

Please share how this access benefits you. Submit a story.

\section{Accessibility}




\title{
Comment on "Method to analyze electromechanical stability of dielectric elastomers" [Appl. Phys. Lett. 91, 061921 (2007)]
}

\author{
Andrew N. Norri:丹 \\ Mechanical and Aerospace Engineering, Rutgers University, Piscataway NJ 08854
}

(Dated: October 24, 2018)

\begin{abstract}
The model of Zhao and Suo can be readily generalized to predict the critical breakdown electric field $E_{c}$ value of elastomers with arbitrary elastic strain energy function. An explicit expression for $E_{c}$ is presented for elastomeric thin films under biaxial strain and comparisons are made with experimental data using a two term Ogden rubber elasticity model. Simplified results for uniaxial and for equi-biaxial stress provide further insight into the findings of Zhao and Suo.
\end{abstract}

PACS numbers: 77.55.+f, 61.41.+e, 77.22.Jp, 83.80.Va, 52.25.Mq

The paper of Zhao and Suo [1] describes a fully nonlinear electromechanical model for the phenomenon of electrical breakdown in thin elastomers. The purpose of this comment is to point out some analytical simplifications which provide further insight into their model, and to provide explicit formulas useful for elastomer design.

The results here stem from the observation that the determinant of the Hessian $\mathbf{H}$ of eq. (4) in [1] may be factored, leading to semi-explicit formulas for the critical values of the electrical and mechanical parameters. It may be checked that the determinant reduces to a quadratic in $z$,

$$
\begin{array}{r}
\operatorname{det} \mathbf{H}=\frac{\mu^{2} \epsilon^{-1}}{\lambda_{1}^{8} \lambda_{2}^{8}}\left[5+3\left(\lambda_{1}^{2}+\lambda_{2}^{2}\right) \lambda_{1}^{2} \lambda_{2}^{2}+\lambda_{1}^{6} \lambda_{2}^{6}\right. \\
\left.+\left[2-\left(\lambda_{1}^{2}+\lambda_{2}^{2}\right) \lambda_{1}^{2} \lambda_{2}^{2}\right] z-3 z^{2}\right]
\end{array}
$$

where the nondimensional parameter $z$ is

$$
z=(\mu \epsilon)^{-1} \widetilde{D}^{2}=\mu^{-1} \epsilon \widetilde{E}^{2} \lambda_{1}^{4} \lambda_{2}^{4}=\mu^{-1} \epsilon E^{2} \lambda_{1}^{2} \lambda_{2}^{2},
$$

and all other notation follows [1]. The roots of the quadratic are real and of opposite sign, so there is a unique positive value of $z$ at which the Hessian is no longer positive definite. It turns out that the same structure of the Hessian is retained for free energy of the form

$$
W\left(\lambda_{1}, \lambda_{2}, \widetilde{D}\right)=U\left(\lambda_{1}, \lambda_{2}\right)+\frac{\widetilde{D}^{2}}{2 \epsilon} \lambda_{1}^{-2} \lambda_{2}^{-2},
$$

where $U\left(\lambda_{1}, \lambda_{2}\right)=\psi\left(\lambda_{1}, \lambda_{2}, \lambda_{1}^{-1} \lambda_{2}^{-1}\right)$. An equation similar to (1) is obtained, and taking the single positive root shows that the critical value of the electric field satisfies

$$
\epsilon E_{c}^{2}=\frac{1}{6}\left(4 \lambda_{1} \lambda_{2} U_{12}-\lambda_{1}^{2} U_{11}-\lambda_{2}^{2} U_{22}+\sqrt{\left(\lambda_{1}^{2} U_{11}+\lambda_{2}^{2} U_{22}-4 \lambda_{1} \lambda_{2} U_{12}\right)^{2}+12 \lambda_{1}^{2} \lambda_{2}^{2}\left(U_{11} U_{22}-U_{12}^{2}\right)}\right),
$$

where $U_{i j}=\partial^{2} U / \partial \lambda_{i} \partial \lambda_{j}$. If the stretches $\lambda_{1}$ and $\lambda_{2}$ are prescribed, then eq. (4) is sufficient to estimate the critical field strength. Otherwise, if the nominal stresses $s_{1}$ and $s_{2}$ are prescribed then the stretches are determined from

$$
s_{j}=U_{j}-\lambda_{j}^{-1} \epsilon E_{c}^{2}, \quad j=1,2 .
$$

Under equi-biaxial strain $\lambda_{1}=\lambda_{2}=\lambda$, eq. (44) becomes

$$
\epsilon E_{c}^{2}=\frac{\lambda^{2}}{3}\left(U_{11}+U_{12}\right),
$$

where the critical value of the stress $s_{1}=s_{2}=s$ is

$$
U_{1}-\frac{1}{3 \lambda}\left(U_{11}+U_{12}\right)=s .
$$

Consider the Ogden model for rubber [2]

$$
\psi\left(\lambda_{1}, \lambda_{2}, \lambda_{3}\right)=\sum_{p=1}^{N} \frac{\mu_{p}}{\alpha_{p}}\left(\lambda_{1}^{\alpha_{p}}+\lambda_{2}^{\alpha_{p}}+\lambda_{3}^{\alpha_{p}}\right),
$$

for which the critical electrical field strength is

$$
\epsilon E_{c}^{2}=\frac{1}{3} \sum_{p=1}^{N} \mu_{p}\left[\left(\alpha_{p}-1\right) \lambda^{\alpha_{p}}+\left(2 \alpha_{p}+1\right) \lambda^{-2 \alpha_{p}}\right] .
$$

If the stress is prescribed then $\lambda$ is given by

$$
\frac{1}{3 \lambda} \sum_{p=1}^{N} \mu_{p}\left[\left(4-\alpha_{p}\right) \lambda^{\alpha_{p}}-\left(4+2 \alpha_{p}\right) \lambda^{-2 \alpha_{p}}\right]=s .
$$


These parameterize the critical electrical and mechanical fields in terms of $\lambda$.

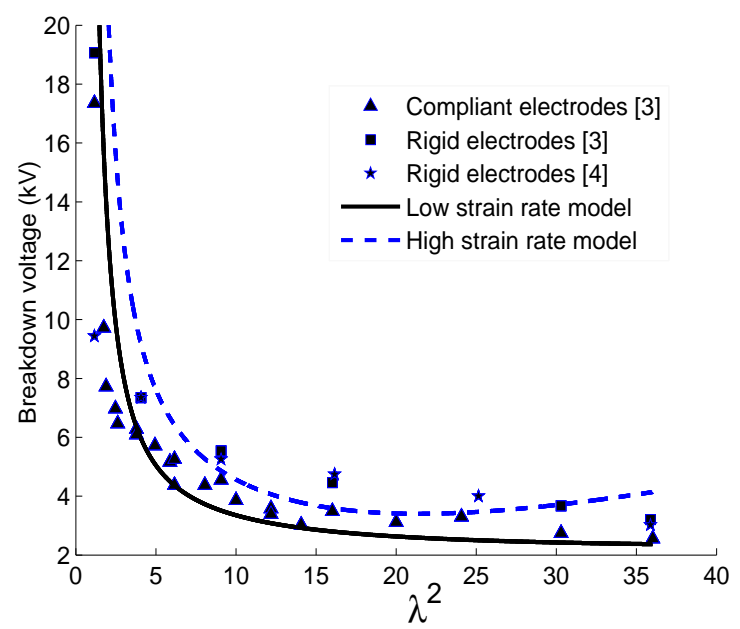

FIG. 1: The data show reported critical breakdown voltages as a function of the equi-biaxial prestrain $\lambda$ for films of VHB 4905/4910 elastomer, from [3, 4] . The curves are the predictions of eq. (9) using the elasticity parameters from [3] with $\epsilon_{d}=12$.

Values of the critical breakdown voltage for the elastomer VHB 4905/4910 have been reported by Plante and Dubowsky [3] and by Kofod et al. [4]. Assuming the Ogden model with $N=2$, Plante and Dubowsky [3] measured values of $\alpha_{1}=1.445(1.450), \alpha_{2}=4.248(8.360)$, $\mu_{1}=43,560(112,200) \mathrm{Pa}, \mu_{2}=117.4(0.1045) \mathrm{Pa}$ for elastomer films of initial thickness $L_{3}=1.5 \mathrm{~mm}$ at low (high) stretch rates. Using these values the critical breakdown voltage $V_{c}=L_{3} \widetilde{E}_{c}$ predicted by eq. (9) is compared with the data of [3, 4] in Figure 1. The material dielectric constant was chosen as $\epsilon_{d}=12$ to fit the curves with the data, where $\epsilon=\epsilon_{d} \epsilon_{0}$ and $\epsilon_{0}=8.85 \times 10^{-12} \mathrm{~F} / \mathrm{m}$ is the free space permittivity. The agreement is reasonable, given that the experiments were not performed in a state of pure equi-biaxial stress.

Some useful explicit results can be determined for the one term Ogden model $\left(N=1, \alpha_{1}, \mu_{1}, \rightarrow \alpha, \mu\right)$. Under equi-biaxial stress the critical stretch satisfies $\lambda \geq \lambda_{c}$ where $\lambda_{c}=((4+2 \alpha) /(4-\alpha))^{1 /(3 \alpha)}$ is the $s=0$ value. This obviously requires that $\alpha<4$. The critical field $E_{c}$ has a unique minimum at $\lambda_{0}=(2(2 \alpha+1) /(\alpha-1))^{1 /(3 \alpha)}$ if $\alpha>1$. Zhao and Suo [1] considered $\alpha=2$, for which $\lambda_{c} \approx 1.26, \lambda_{0} \approx 1.47$ and the minimum value of $\sqrt{\frac{\epsilon}{\mu}} E_{c}$ is 1.038 .

Finally, we note that the neo-Hookean constitutive model of Zhao and Suo [1] is apparently unique among the $N=1$ Ogden models in that it yields a simple formula for uniaxial stress. Thus, eq. (5) with $N=1, \alpha=2$ for $j=2$ and $s_{2}=0$ yields the relation $\lambda_{1}^{2}=3 \lambda_{2}^{2} /\left(\lambda_{2}^{6}-1\right)$ between the stretches. Hence, we can parameterize the critical values in terms of $1<\lambda_{2} \leq \lambda_{c} \approx 1.26$ :

$$
\begin{aligned}
\sqrt{\frac{\epsilon}{\mu}} E_{c} & =\left(\frac{2}{3} \lambda_{2}^{2}+\frac{1}{3} \lambda_{2}^{-4}\right)^{1 / 2} \\
\frac{s_{1}}{\mu} & =\frac{\lambda_{2}}{\sqrt{3}} \frac{\left(4-\lambda_{2}^{6}\right)}{\sqrt{\lambda_{2}^{6}-1}} .
\end{aligned}
$$

In this case $E_{c}$ is a monotonically decreasing function of the stress $s_{1}$, and $\sqrt{\frac{\epsilon}{\mu}} E_{c} \rightarrow 1$ in the limit of large uniaxial stress. Figure 3(b) in [1] indicates that this is the smallest achievable value of the critical electric field strength. Generalization of the formulas (11) to $\alpha \neq 2$ is possible but far more complicated.

In summary, the model of Zhao and Suo readily generalizes to arbitrary elastic strain energy. The explicit results reported here, such as eq. (4), can be used to compare different elastic constitutive models, and should be helpful in the design of elastomeric actuators.
[1] X. Zhao and Z. Suo, Appl. Phys. Lett. 91, 061921 (2007).

[2] R. W. Ogden, Non-Linear Elastic Deformations (Ellis Horwood, 1984).

[3] J.-S. Plante and S. Dubowsky, Int. J. Solids Struct. 43, 7727 (2006).
[4] G. Kofod, R. Kornbluh, R. Pelrine, and P. SommerLarsen, in Proceedings of Smart Structures and Materials 2001: Electroactive Polymer Actuators and Devices (EAPAD) (SPIE, San Diego, CA, 2001), vol. 4329, pp. 141147. 